\title{
NUMERICAL METHODS FOR THE NONLINEAR SCHRÖDINGER EQUATION WITH NONZERO FAR-FIELD CONDITIONS*
}

\author{
WEIZHU $\mathrm{BAO}^{\dagger}$
}

Dedicated to Professor Georege Papanicolaou on the occasion of his 60th birthday

Abstract. In this paper we present numerical methods for the nonlinear Schrödinger equations (NLS) in the semiclassical regimes:

$$
i \varepsilon u_{t}^{\varepsilon}=-\frac{\varepsilon^{2}}{2} \Delta u^{\varepsilon}+V(\mathbf{x}) u^{\varepsilon}+f\left(\left|u^{\varepsilon}\right|^{2}\right) u^{\varepsilon}, \quad \mathbf{x} \in \mathbb{R}^{d},
$$

with nonzero far-field conditions. A time-splitting cosine-spectral (TS-Cosine) method is presented when the nonzero far-field conditions are or can be reduced to homogeneous Neumann conditions, a time-splitting Chebyshev-spectral (TS-Chebyshev) method is proposed for more general nonzero farfield conditions, and an efficient and accurate numerical method in which we use polar coordinates to properly match the nonzero far-field conditions is presented for computing dynamics of quantized vortex lattice of NLS in two dimensions (2D). All the methods are explicit, unconditionally stable and time reversible. Furthermore, TS-Cosine is time-transverse invariant and conserves the position density, where TS-Chebyshev can deal with more general nonzero far-field conditions. Extensive numerical tests are presented for linear constant/harmonic oscillator potential, defocusing nonlinearity of NLS to study the $\varepsilon$-resolution of the methods. Our numerical tests suggest the following 'optimal' $\varepsilon$-resolution of the methods for obtaining 'correct' physical observables in the semi-classical regimes: time step $k$-independent of $\varepsilon$ and mesh size $h=O(\varepsilon)$ for linear case; $k=O(\varepsilon)$ and $h=O(\varepsilon)$ for defocusing nonlinear case. The methods are applied to study numerically the semiclassical limits of NLS in $1 \mathrm{D}$ and the dynamics of quantized vortex lattice of NLS in 2D with nonzero far-field conditions.

Key words. Nonlinear Schrödinger equation, nonzero far-field conditions, semiclassical regime, semiclassical limit, $\varepsilon$-resolution, quantized vortex lattice

AMS subject classifications. 65M12, 65M70, 65T40, 81Q05

1. Introduction. The specific problem we study numerically in this paper is that of the nonlinear Schrödinger equation (NLS) with a small (scaled) Planck constant $\varepsilon(0<\varepsilon \ll 1)$ given by $[23,28,11,26,20]$ :

$$
i \varepsilon u_{t}^{\varepsilon}=-\frac{\varepsilon^{2}}{2} \Delta u^{\varepsilon}+V(\mathbf{x}) u^{\varepsilon}+f\left(\left|u^{\varepsilon}\right|^{2}\right) u^{\varepsilon}, \quad t>0, \quad \mathbf{x} \in \mathbb{R}^{d}
$$

with nonzero far-field conditions and the initial condition

$$
u^{\varepsilon}(\mathbf{x}, t=0)=u_{0}^{\varepsilon}(\mathbf{x}), \quad \mathbf{x} \in \mathbb{R}^{d}
$$

In this problem, $\mathbf{x}=\left(x_{1}, \cdots, x_{d}\right)^{T}$ is the Cartesian coordinate, $u^{\varepsilon}=u^{\varepsilon}(\mathbf{x}, t)$ is the complex wave function, $V=V(\mathbf{x})$ is a given real-valued potential, $f$ is a realvalued smooth function. Three typical types of nonzero far-field conditions are used in literatures. The first one is the homogeneous Neumann far-field condition $[23,10]$

$$
\frac{\partial u^{\varepsilon}(\mathbf{x}, t)}{\partial x_{j}} \rightarrow 0, \quad x_{j} \rightarrow \pm \infty, \quad j=1, \cdots, d
$$

\footnotetext{
*Received July 28, 2004; accepted for publication April 26, 2005.

${ }^{\dagger}$ Department of Computational Science, National University of Singapore, Singapore 117543 (bao@cz3.nus.edu.sg).
} 
The second one reads $[16,17]$

$$
u^{\varepsilon}(\mathbf{x}, t) \rightarrow A_{\infty} \exp \left(\frac{i \mathbf{x} \cdot \mathbf{S}_{\infty}}{\varepsilon|\mathbf{x}|}\right), \quad \text { as }|\mathbf{x}| \rightarrow \infty
$$

where $\mathbf{S}_{\infty}=\left(S_{1 \infty}, \cdots, S_{d \infty}\right)^{T}$ with $A_{\infty}, S_{1 \infty}, \cdots, S_{d \infty}$ constants, $|\mathbf{x}|=$ $\sqrt{x_{1}^{2}+\cdots+x_{d}^{2}}, \mathbf{x} \cdot \mathbf{S}_{\infty}=x_{1} S_{1 \infty}+\cdots+x_{d} S_{d \infty}$, and the third one which is usually used for studying vortex motion of NLS $[23,28,10]$ in $2 \mathrm{D}$ is

$$
u^{\varepsilon}\left(x_{1}, x_{2}, t\right) \rightarrow A_{\infty} \exp (i m \theta), \quad \text { as } r=|\mathbf{x}|=\sqrt{x_{1}^{2}+x_{2}^{2}} \rightarrow \infty,
$$

here $(r, \theta)$ is the polar coordinate, $A_{\infty}$ is a constant and $m$ is an integer. For the boundedness of the energy functional of the NLS (1.1) [21], we assume

$$
\left[V(\mathbf{x})+f\left(A_{\infty}^{2}\right)\right] A_{\infty}=0, \quad \text { as }|\mathbf{x}| \rightarrow \infty .
$$

In quantum mechanics, the wave function is an auxiliary quantity used to compute the primary physical quantities such as the position density

$$
\rho^{\varepsilon}(\mathbf{x}, t)=\left|u^{\varepsilon}(\mathbf{x}, t)\right|^{2}
$$

and the current density

$$
J^{\varepsilon}(\mathbf{x}, t)=\varepsilon \operatorname{Im}\left(\overline{u^{\varepsilon}(\mathbf{x}, t)} \nabla u^{\varepsilon}(\mathbf{x}, t)\right)=\frac{\varepsilon}{2 i}\left(\overline{u^{\varepsilon}} \nabla u^{\varepsilon}-u^{\varepsilon} \nabla \overline{u^{\varepsilon}}\right),
$$

where "—" denotes complex conjugation.

The general form of (1.1) covers many nonlinear Schrödinger equations arising in various different applications. For example, when $f(\rho) \equiv 0, A_{\infty}=0$ in (1.4), it reduces to the linear Schrödinger equation; when $V(\mathbf{x}) \equiv 0, f(\rho)=\beta_{\varepsilon} \rho, A_{\infty}=0.0$ in (1.4), it is the cubic NLS (called the focusing NLS if $\beta_{\varepsilon}<0$ and the defocusing NLS if $\beta_{\varepsilon}>0$ [21]); when $V(\mathbf{x}) \equiv 0, f(\rho)=\rho-1, A_{\infty}=1.0$ in (1.4), it corresponds to the propagation of a wave beam in a defocusing medium $[32,33]$; when $V(\mathbf{x}) \equiv 0$, $f(\rho)=\rho-1, A_{\infty}=1.0$ in (1.5), it corresponds to a vortex motion of NLS in 2D $[28,23,10]$.

It is well known that the equation (1.1) propagates oscillations in space and time, preventing $u^{\varepsilon}$ from converging strongly as $\varepsilon \rightarrow 0$. Much progress has been made recently in analytical understanding semiclassical limits of the linear Schödinger equation (i.e. $f(\rho) \equiv 0$ in (1.1)), particularly by the introduction of tools from microlocal analysis, such as defect measures [13], H-measures [30], and Wigner measures $[12,14,24]$. These techniques have not been successfully extended to the semiclassical limit of the NLS, except that the 1D defocusing (cubically) NLS (1.1) was solved by using techniques of inverse scattering $[16,17]$. Thus it is a very interesting problem to study the semiclassical limit of NLS numerically.

The oscillatory nature of solutions of the Schrödinger equation with small $\varepsilon$ provides severe numerical burdens. In [25, 26], Markowich et al. studied the finite difference approximation of the linear Schrödinger equation with small $\varepsilon$ and zero far-field condition. Their results show that, for the best combination of the time and space discretizations, one needs the following constraints in order to guarantee good approximations to all (smooth) observables for $\varepsilon$ small [25, 26]: mesh size $h=o(\varepsilon)$ and time step $k=o(\varepsilon)$. The same or more severe meshing constraint is required by the finite difference approximation of the NLS in the semi-classical regime with zero 
far-field condition, i.e. $A_{\infty}=0$ in (1.4). Failure to satisfy these conditions leads to wrong numerical observables. Recently, Bao et al. studied the time-splitting Fourierspectral (TS-Fourier) [2,3] and sine-spectral (TS-Sine) [5] methods for the NLS with zero far-field condition and applied them successfully to simulate Bose-Einstein condensation [4], in this case the periodic or homogeneous Dirichlet boundary condition can be applied. Because the TS-Fourier and TS-Sine methods are unconditionally stable, time reversible and time-transverse invariant, spectral accuracy in space and conserves the position density, they observed 'optimal' $\varepsilon$-resolution for obtaining 'correct' physical observables: time step $k$-independent of $\varepsilon$ and mesh size $h=O(\varepsilon)$ for linear case; $k=O(\varepsilon)$ and $h=O(\varepsilon)$ for defocusing nonlinear case, which is much more better than the finite difference methods $[2,3]$. Unfortunely, the TS-Fourier and TS-Sine methods can not be applied to approximate NLS (1.1) with nonzero far-field conditions (1.3), or (1.4), or (1.5).

In this paper some new numerical methods are presented for the NLS (1.1) with non-zero far-field conditions (1.3)-(1.5). The first one is the time-splitting cosinespectral (TS-Cosine) method which can deal with homogeneous Neumann far-field condition (1.3), and zero, i.e. $A_{\infty}=0$, or nonzero, i.e. $A_{\infty} \neq 0$, far-field condition (1.4) which can be reduced to (1.3). This method is unconditionally stable, time reversible, time-transverse invariant and conserves the position density. The second one is the time-splitting Chebyshev-spectral (TS-Chebyshev) method which can deal with more general nonzero far-field conditions, e.g. (1.5). This method is time reversible and time-transverse invariant when it is applied to zero far-field condition, i.e $A_{\infty}=0$ in (1.5). Our goal to propose these two methods is to deal with nonzero far-field condition, i.e. $A_{\infty} \neq 0$ in (1.4) and (1.5), and understand the $\varepsilon$-resolution capacity of the methods for NLS as well as investigate numerically the semiclassical limit of the NLS with nonzero far-field conditions. Our numerical experiments suggest the following 'optimal' $\varepsilon$-resolution of the methods for obtaining the correct observables: $k$-independent of $\varepsilon$ and $h=O(\varepsilon)$ for linear Schrödinger equation; $k=O(\varepsilon)$ and $h=O(\varepsilon)$ for cubic defocusing NLS. The third one is an efficient and accurate numerical method for the NLS (1.1) in 2D with the nonzero far-field conditions (1.5). Due to the oscillatory nature in the transverse direction of the far-field condition (1.5), in order to study effectively vortex dynamics of NLS in 2D, especially when $|m| \gg 1$ in (1.5), an efficient and accurate numerical method is one of the key issues. To our knowledge, currently the numerical methods proposed in the literature for studying vortex dynamics of NLS in 2D with the nonzero far-field conditions (1.5) remain very limited, and they usually are low-order methods. Thus it is of great interests to develop an efficient, accurate and unconditionally stable numerical method for the NLS in 2D with the nonzero far-field conditions (1.5). Such a numerical method is proposed here and it is applied to study dynamics of quantized vortex lattice of NLS in 2D with nonzero far-field conditions. The key features of this method are based on: (i) the application of a time-splitting technique for decoupling the nonlinearity in the NLS; (ii) the adoption of polar coordinates to match the oscillatory nature in the transverse direction of the far-field conditions (1.5); and (iii) the utilization of Fourier pseudo-spectral discretization in the transverse direction and a second order finite difference discretization in the radial direction.

The paper is organized as follows. In section 2 we present the TS-Cosine approximations of the NLS (1.1) with homogeneous Neumann far-field conditions (1.3). In section 3 we propose the TS-Chebyshev discretizations of the NLS (1.1) with general far-field conditions. In section 4 we present an efficient and accurate method for 
computing dynamics of quantized vortex lattice of NLS in 2D with non-zero far-field condition (1.5). In section 5 numerical tests for linear/nonlinear Schrödinger equation with zero/nonzero $A_{\infty}$ and $S_{\infty}$ in (1.4) or (1.5) are presented. In section 6 some conclusions are drawn.

2. Time-splitting cosine-spectral approximations. In this section we present the TS-Cosine approximations of the NLS (1.1) with homogeneous Neumann far-field condition (1.3) or nonzero far-field condition, e.g. (1.4), which can be reduced to (1.3). We suppose the initial data (1.2) satisfies decay conditions (1.3) or (1.4) sufficiently rapidly, say, faster than any power of $\mathbf{x}$. Here we shall introduce the method in 1D. Generalizations to higher dimension are straightforward for tensor product grids and the results remain valid without modifications. In $1 \mathrm{D}$, we consider

$$
\begin{aligned}
& i \varepsilon u_{t}^{\varepsilon}=-\frac{\varepsilon^{2}}{2} u_{x x}^{\varepsilon}+V(x) u^{\varepsilon}+f\left(\left|u^{\varepsilon}\right|^{2}\right) u^{\varepsilon}, \quad a<x<b, \quad t>0, \\
& \partial_{x} u^{\varepsilon}(a, t)=\partial_{x} u^{\varepsilon}(b, t)=0, \quad t \geq 0, \\
& u^{\varepsilon}(x, t=0)=u_{0}^{\varepsilon}(x), \quad a \leq x \leq b .
\end{aligned}
$$

We choose the spatial mesh size $h=\Delta x>0$ with $h=(b-a) / M$ for $M$ an even positive integer, the time step size $k=\Delta t>0$ and let the grid points and time steps be

$$
x_{j}:=a+(j+1 / 2) h, \quad j=0,1, \cdots, M-1, \quad t_{n}:=n k, \quad n=0,1,2, \cdots .
$$

Let $U_{j}^{\varepsilon, n}$ be the approximation of $u^{\varepsilon}\left(x_{j}, t_{n}\right)$ and $U^{\varepsilon, n}$ be the solution vector at time $t=t_{n}=n k$ with components $U_{j}^{\varepsilon, n}$.

Time-splitting cosine-spectral (TS-Cosine) method. From time $t=t_{n}$ to time $t=t_{n+1}$, the Schrödinger equation (2.1) is solved in two steps. One solves

$$
i \varepsilon u_{t}^{\varepsilon}=-\frac{\varepsilon^{2}}{2} u_{x x}^{\varepsilon}
$$

with the boundary condition (2.2) for one time step, followed by solving

$$
i \varepsilon u_{t}^{\varepsilon}(x, t)=V(x) u^{\varepsilon}(x, t)+f\left(\left|u^{\varepsilon}(x, t)\right|^{2}\right) u^{\varepsilon}(x, t),
$$

again for one time step. Equation (2.4) will be discretized in space by the cosinespectral method and integrated in time exactly. For $t \in\left[t_{n}, t_{n+1}\right]$, the ODE (2.5) leaves $|u|$ invariant in $t[2]$ and therefore becomes

$$
i \varepsilon u_{t}^{\varepsilon}(x, t)=V(x) u^{\varepsilon}(x, t)+f\left(\left|u^{\varepsilon}\left(x, t_{n}\right)\right|^{2}\right) u^{\varepsilon}(x, t)
$$

and thus can be integrated exactly. The solution of (2.6) is given by

$$
u^{\varepsilon}(x, t)=e^{-i\left[V(x)+f\left(\left|u^{\varepsilon}\left(x, t_{n}\right)\right|^{2}\right)\right]\left(t-t_{n}\right) / \varepsilon} u^{\varepsilon}\left(x, t_{n}\right), \quad t \in\left[t_{n}, t_{n+1}\right] .
$$

From time $t=t_{n}$ to $t=t_{n+1}$, we combine the splitting steps via the standard second-order Strang splitting [29] and obtain a second-order time-splitting cosinespectral method (TS-Cosine2) for the Schrödinger equation (3.1). The detailed method is given by

$$
\begin{aligned}
& U_{j}^{*}=e^{-i\left[V\left(x_{j}\right)+f\left(\left|U_{j}^{\varepsilon, n}\right|^{2}\right)\right] k /(2 \varepsilon)} U_{j}^{\varepsilon, n}, \\
& U_{j}^{* *}=\frac{2}{M} \sum_{l=0}^{M-1} \alpha_{l} e^{-i \varepsilon k \mu_{l}^{2} / 2} \widehat{\left(U^{*}\right)_{l}} \cos \left(\frac{(2 j+1) l \pi}{2 M}\right), \\
& U_{j}^{\varepsilon, n+1}=e^{-i\left[V\left(x_{j}\right)+f\left(\left|U_{j}^{* *}\right|^{2}\right)\right] k /(2 \varepsilon)} U_{j}^{* *}, \quad j=0,1, \cdots, M-1,
\end{aligned}
$$


where $\widehat{U}_{l}$, the cosine-transform coefficients of a complex vector $U=\left(U_{0}, U_{1}, \cdots\right.$, $\left.U_{M-1}\right)$, are defined as

$$
\begin{aligned}
& \mu_{l}=\frac{\pi l}{b-a}, \quad l=0,1, \cdots, M, \quad \alpha_{l}= \begin{cases}1 / \sqrt{2}, & l=0, M \\
1, & l=1, \cdots, M-1 .\end{cases} \\
& \widehat{U}_{l}=\alpha_{l} \sum_{j=0}^{M-1} U_{j} \cos \left(\mu_{l}\left(x_{j}-a\right)\right)=\alpha_{l} \sum_{j=0}^{M-1} U_{j} \cos \left(\frac{(2 j+1) l \pi}{2 M}\right), 0 \leq l<M,
\end{aligned}
$$

with

$$
U_{j}^{\varepsilon, 0}=u^{\varepsilon}\left(x_{j}, 0\right)=u_{0}^{\varepsilon}\left(x_{j}\right), \quad j=0,1, \cdots, M-1 .
$$

Note that the only time discretization error of TS-Cosine2 is the splitting error, which is second order in $k$ for any fixed $\varepsilon>0$.

From time $t=t_{n}$ to $t=t_{n+1}$, we combine the splitting steps via the fourth-order split-step method [34] and obtain a fourth-order time-splitting cosine-spectral (TSCosine4) method for the Schrödinger equation (2.1). The detailed method is given by

$$
\begin{aligned}
U_{j}^{(1)} & =e^{-i 2 w_{1} k\left[V\left(x_{j}\right)+f\left(\left|U_{j}^{\varepsilon, n}\right|^{2}\right)\right] / \varepsilon} U_{j}^{\varepsilon, n}, \\
U_{j}^{(2)} & =\frac{2}{M} \sum_{l=0}^{M-1} \alpha_{l} e^{-i \varepsilon w_{2} k \mu_{l}^{2}} \widehat{\left(U^{(1)}\right)_{l}} \cos \left(\frac{(2 j+1) l \pi}{2 M}\right), \\
U_{j}^{(3)} & =e^{-i 2 w_{3} k\left[V\left(x_{j}\right)+f\left(\left|U_{j}^{(2)}\right|^{2}\right)\right] / \varepsilon} U_{j}^{(2)}, \\
U_{j}^{(4)} & =\frac{2}{M} \sum_{l=0}^{M-1} \alpha_{l} e^{-i \varepsilon w_{4} k \mu_{l}^{2}} \widehat{\left(U^{(3)}\right)_{l}} \cos \left(\frac{(2 j+1) l \pi}{2 M}\right), \\
U_{j}^{(5)} & =e^{-i 2 w_{3} k\left[V\left(x_{j}\right)+f\left(\left|U_{j}^{(4)}\right|^{2}\right)\right] / \varepsilon} U_{j}^{(4)}, \\
U_{j}^{(6)} & =\frac{2}{M} \sum_{l=0}^{M-1} \alpha_{l} e^{-i \varepsilon w_{2} k \mu_{l}^{2}} \widehat{\left(U^{(5)}\right)_{l}} \cos \left(\frac{(2 j+1) l \pi}{2 M}\right), \\
U_{j}^{\varepsilon, n+1} & =e^{-i 2 w_{1} k\left[V\left(x_{j}\right)+f\left(\left|U_{j}^{(6)}\right|^{2}\right)\right] / \varepsilon} U_{j}^{(6)}, \quad j=0,1, \cdots, M-1 ;
\end{aligned}
$$

with [34]

$$
\begin{aligned}
& w_{1}=0.33780179798991440851, \quad w_{2}=0.67560359597982881702, \\
& w_{3}=-0.08780179798991440851, \quad w_{4}=-0.85120719795965763405 .
\end{aligned}
$$

Again the only time discretization error of TS-Cosines4 is the splitting error, which is now fourth order in $k$ for any fixed $\varepsilon>0$.

The schemes TS-Cosine 4 and TS-Cosine 2 are explicit, time reversible, just as the IVP for the NLS. Also, a main advantage of the two methods is their time-transverse invariance, just as it holds for the NLS itself. If a constant $\alpha$ is added to the potential $V$, then the discrete wave functions $U_{j}^{\varepsilon, n+1}$ obtained from TS-Cosine4 or TS-Cosine2 get multiplied by the phase factor $e^{-i \alpha(n+1) k / \varepsilon}$, which leaves the discrete quadratic observables unchanged. This property does not hold for finite difference schemes $[25,26]$. 
REMARK 2.1. If $V(x) \equiv V=$ constant and $f(\rho) \equiv 0$ in (2.1), then all the time steps in TS-Cosine2 or TS-Cosine4 can be combined and the methods can be written simply as a one step method:

$$
U_{j}^{\varepsilon, n}=\frac{2}{M} \sum_{l=0}^{M-1} \alpha_{l} e^{-i\left(\varepsilon \mu_{l}^{2} / 2+V / \varepsilon\right) t_{n}} \widehat{\left(U^{\varepsilon, 0}\right)_{l}} \cos \left(\frac{(2 j+1) l \pi}{2 M}\right), \quad j=0,1, \cdots, M-1 .
$$

This is the same as discretizing the second order space derivative in (2.1) by the cosine-spectral method, and then solving the resulting ODE system exactly to $t=t_{n}$. Therefore no time discretization error is introduced and the only error is the cosinespectral error of the spatial derivative.

Let $U=\left(U_{0}, U_{1}, \cdots, U_{M-1}\right)^{T}$ and let $\|\cdot\|_{l^{2}}$ be the usual discrete $l^{2}$-norm on the interval $(a, b)$, i.e.

$$
\|U\|_{l^{2}}=\sqrt{\frac{b-a}{M} \sum_{j=0}^{M-1}\left|U_{j}\right|^{2}}
$$

For the stability of the time-splitting cosine-spectral approximations TS-Cosine 4 and TS-Cosine2, we have the following lemma, which shows that the total charge is conserved.

LEMma 2.1. The schemes TS-Cosine4 (2.12) and TS-Cosine2 (2.8) are unconditionally stable. In fact, for every mesh size $h>0$ and time step $k>0$,

$$
\left\|U^{\varepsilon, n}\right\|_{l^{2}}=\left\|U^{\varepsilon, 0}\right\|_{l^{2}}=\left\|u_{0}^{\varepsilon}\right\|_{l^{2}}, \quad n=1,2, \cdots .
$$

Proof. Follows the line of the analogous result for the linear Schrödinger by timesplitting Fourier-spectral approximation in [2].

3. Time-splitting Chebyshev-spectral method. In this section we present time-splitting Chebyshev-spectral approximations of the NLS (1.1) with more general far-field conditions, e.g. (1.5). Again here we shall introduce the method in 1D. Generalizations to higher dimension are straightforward for tensor product grids and the results remain valid without modifications. In 1D, we consider

$$
\begin{aligned}
& i \varepsilon u_{t}^{\varepsilon}=-\frac{\varepsilon^{2}}{2} u_{x x}^{\varepsilon}+V(x) u^{\varepsilon}+f\left(\left|u^{\varepsilon}\right|^{2}\right) u^{\varepsilon}, \quad a<x<b, \quad t>0, \\
& u^{\varepsilon}(a, t)=g_{1}, \quad u^{\varepsilon}(b, t)=g_{2}, \quad t \geq 0, \\
& u^{\varepsilon}(x, t=0)=u_{0}^{\varepsilon}(x), \quad a \leq x \leq b
\end{aligned}
$$

where $g_{1}$ and $g_{2}$ are two given complex numbers. The numerical methods in this section can be generalized straightforward to problems with boundary conditions other than (3.2).

Let the grid points be

$$
x_{j}:=\frac{b-a}{2} \cos \frac{j \pi}{M}+\frac{b+a}{2}, \quad j=0,1, \cdots, M .
$$


Here the spatial grid points are chosen as the Chebyshev-Gauss-Lobatto interpolation points for simplicity $[15,7]$. The other types of interpolation points, e.g. ChebyshevGauss or Chebyshev-Gauss-Radau type [15, 7], can also be used with few modifications. Let $U_{j}^{\varepsilon, n}$ be the approximation of $u^{\varepsilon}\left(x_{j}, t_{n}\right)$ and $U^{\varepsilon, n}$ be the solution vector at time $t=t_{n}=n k$ with components $U_{j}^{\varepsilon, n}$.

Time-splitting Chebyshev-spectral (TS-Chebyshev) method. From time $t=t_{n}$ to $t=t_{n+1}$, the NLS equation (3.1) is solved in two steps. One solves first

$$
i \varepsilon u_{t}^{\varepsilon}=-\frac{\varepsilon^{2}}{2} u_{x x}^{\varepsilon}
$$

with the boundary condition (3.2) for one time step (of length $k$ ), followed by solving

$$
i \varepsilon u_{t}^{\varepsilon}=V(x) u^{\varepsilon}+f\left(\left|u^{\varepsilon}\right|^{2}\right) u^{\varepsilon},
$$

for the same time step. The solution of the ODE (3.5) is given explicitly in (2.7). Equation (3.4) with the boundary condition (3.2) will be discretized in space by the Chebyshev-spectral tau method $[15,7]$ and integrated in time exactly by applying a diagonalization technique for the ODE system in phase space. Let

$$
u_{M}^{\varepsilon}(x, t)=\sum_{m=0}^{M} a_{m}(t) T_{m}\left(\frac{x-(b+a) / 2}{(b-a) / 2}\right), \quad a \leq x \leq b
$$

where $T_{m}(x)$ is the $m$-th Chebyshev polynomial [15, 7]. Plugging (3.6) into (3.4) and (3.2), one obtains

$$
\begin{aligned}
& i \varepsilon a_{m}^{\prime}(t)+\frac{2 \varepsilon^{2}}{(b-a)^{2} c_{m}} \sum_{\substack{p=m+2 \\
p+m \text { even }}}^{M} p\left(p^{2}-m^{2}\right) a_{p}(t)=0,0 \leq m \leq M-2, \\
& \sum_{m=0}^{M} a_{m}(t)=g_{1}, \quad \sum_{m=0}^{M}(-1)^{m} a_{m}(t)=g_{2} ;
\end{aligned}
$$

where

$$
c_{0}=2, \quad c_{m}=1 \text { for } m>0 .
$$

Let

$$
\mathbf{a}(t)=\left[\begin{array}{c}
a_{0}(t) \\
a_{1}(t) \\
a_{2}(t) \\
\vdots \\
a_{M-2}(t)
\end{array}\right], \quad \mathbf{f}=\frac{i \varepsilon}{(b-a)^{2}}\left[\begin{array}{c}
\frac{M\left(M^{2}-0^{2}\right)}{c_{0}}\left(g_{1}+g_{2}\right) \\
\frac{i(M-1)\left((M-1)^{2}-1^{2}\right)}{c_{1}}\left(g_{1}-g_{2}\right) \\
\frac{M\left(M^{2}-2^{2}\right)}{c_{2}}\left(g_{1}+g_{2}\right) \\
\vdots \\
\frac{M\left(M^{2}-(M-2)^{2}\right)}{c_{M-2}}\left(g_{1}+g_{2}\right)
\end{array}\right] .
$$

Then the equations (3.7) and (3.8) can be written as

$$
\frac{d \mathbf{a}(t)}{d t}=\frac{2 i \varepsilon}{(b-a)^{2}} T \mathbf{a}(t)+\mathbf{f},
$$


where $T=\left(t_{j k}\right)$ is an $(M-1) \times(M-1)$ matrix with entries

$$
t_{j k}=\frac{1}{c_{j}} \begin{cases}-M\left(M^{2}-j^{2}\right), & 0 \leq k \leq j, j, k \text { even }, \\ k\left(k^{2}-j^{2}\right)-M\left(M^{2}-j^{2}\right), & j+2 \leq k \leq N-2, j, k \text { even } \\ -(M-1)\left((M-1)^{2}-j^{2}\right), & 1 \leq k \leq j, j, k \text { odd }, \\ k\left(k^{2}-j^{2}\right)-(M-1)\left((M-1)^{2}-j^{2}\right), & j+2 \leq k \leq N-2, j, k \text { odd } \\ 0, & \text { else, } \\ j, k=0,1,2, \cdots, M-2 .\end{cases}
$$

The matrix $T$ has $M-1$ distinct negative eigenvalues [15]. Thus it is diagonalizable, i.e. there is an invertible matrix $P$ and a diagonal matrix $D$ such that

$$
T=P D P^{-1} \text {. }
$$

Let

$$
\mathbf{b}(t)=P^{-1} \mathbf{a}(t) .
$$

Multiplying (3.10) by the matrix $P^{-1}$, noting (3.12), one obtains

$$
\frac{d \mathbf{b}(t)}{d t}=\frac{2 i \varepsilon}{(b-a)^{2}} D \mathbf{b}(t)+P^{-1} \mathbf{f} .
$$

The above ODE system can be integrated exactly, i.e.

$$
\mathbf{b}(t)=\frac{i(b-a)^{2}}{2 \varepsilon} D^{-1} P^{-1} \mathbf{f}+\exp \left(\frac{2 \varepsilon i\left(t-t_{n}\right)}{(b-a)^{2}} D\right) \mathbf{c}, \quad t \in\left[t_{n}, t_{n+1}\right],
$$

where $\mathbf{c}$ is a constant vector to be determined. Multiplying (3.14) by the matrix $P$, noting (3.12) and (3.11), one gets

$$
\begin{aligned}
\mathbf{a}(t) & =P \mathbf{b}(t)=\frac{i(b-a)^{2}}{2 \varepsilon} P D^{-1} P^{-1} \mathbf{f}+P \exp \left(\frac{2 \varepsilon i\left(t-t_{n}\right)}{(b-a)^{2}} D\right) \mathbf{c} \\
& =\frac{i(b-a)^{2}}{2 \varepsilon} T^{-1} \mathbf{f}+P \exp \left(\frac{2 \varepsilon i\left(t-t_{n}\right)}{(b-a)^{2}} D\right) \mathbf{c} .
\end{aligned}
$$

Choosing $t=t_{n}$ in (3.15), one has

$$
\mathbf{a}\left(t_{n}\right)=\frac{i(b-a)^{2}}{2 \varepsilon} T^{-1} \mathbf{f}+P \mathbf{c} .
$$

Solving the above equation (3.16) for $\mathbf{c}$, one gets

$$
\mathbf{c}=P^{-1} \mathbf{a}\left(t_{n}\right)-\frac{i(b-a)^{2}}{2 \varepsilon} P^{-1} T^{-1} \mathbf{f} .
$$

Substituting (3.17) into (3.15), one obtains

$$
\begin{aligned}
\mathbf{a}(t)= & \frac{i(b-a)^{2}}{2 \varepsilon} T^{-1} \mathbf{f}+P \exp \left(\frac{2 i \varepsilon\left(t-t_{n}\right)}{(b-a)^{2}} D\right)\left[P^{-1} \mathbf{a}\left(t_{n}\right)-\frac{i(b-a)^{2}}{2 \varepsilon} P^{-1} T^{-1} \mathbf{f}\right] \\
= & \frac{i(b-a)^{2}}{2 \varepsilon}\left[I-P \exp \left(\frac{2 i \varepsilon\left(t-t_{n}\right)}{(b-a)^{2}} D\right) P^{-1}\right] T^{-1} \mathbf{f} \\
& +P \exp \left(\frac{2 i \varepsilon\left(t-t_{n}\right)}{(b-a)^{2}} D\right) P^{-1} \mathbf{a}\left(t_{n}\right) \\
\equiv & Q\left(t-t_{n}\right) \mathbf{a}\left(t_{n}\right)+\mathbf{g}\left(t-t_{n}\right), \quad t \in\left[t_{n}, t_{n+1}\right]
\end{aligned}
$$


where

$$
Q(\tau)=P \exp \left(\frac{2 i \varepsilon \tau}{(b-a)^{2}} D\right) P^{-1}, \quad \mathbf{g}(\tau)=\frac{i(b-a)^{2}}{2 \varepsilon}[I-Q(\tau)] T^{-1} \mathbf{f},
$$

with $I$ the $(M-1) \times(M-1)$ identity matrix.

From time $t=t_{n}$ to $t=t_{n+1}$, notice (2.7), (3.8), (3.6) and (3.18), we combine the split steps via the standard Strang splitting [29] and obtain a second-order timesplitting Chebyshev (TS-Chebyshev2) method:

$$
\begin{aligned}
& U_{j}^{\varepsilon, *}=e^{-i\left(V\left(x_{j}\right)+f\left(\left|U_{j}^{\varepsilon, n}\right|^{2}\right)\right) k / 2 \varepsilon} U_{j}^{\varepsilon, n} ; \\
& a_{l}^{\varepsilon, *}=\frac{1}{\gamma_{l}} \sum_{m=0}^{M} U_{m}^{\varepsilon, *} \omega_{m} T_{l}\left(\frac{x_{m}-(b+a) / 2}{(b-a) / 2}\right), \quad 0 \leq l \leq M, \\
& \mathbf{a}^{\varepsilon, * *}=Q(k) \mathbf{a}^{\varepsilon, *}+\mathbf{g}(k), \\
& a_{M-1}^{\varepsilon, * *}=\frac{g_{1}-g_{2}}{2}-\sum_{\substack{m=1 \\
m \text { odd }}}^{M-2} a_{m}^{\varepsilon, * *}, \quad a_{M}^{\varepsilon, * *}=\frac{g_{1}+g_{2}}{2}-\sum_{\substack{m=0 \\
m \text { even }}}^{M-2} a_{m}^{\varepsilon, * *}, \\
& U_{j}^{\varepsilon, * *}=\sum_{m=0}^{M} a_{m}^{\varepsilon, * *} T_{m}\left(\frac{x_{j}-(b+a) / 2}{(b-a) / 2}\right), \quad 0 \leq j \leq M, \\
& U_{j}^{\varepsilon, n+1}=e^{-i\left(V\left(x_{j}\right)+f\left(\left|U_{j}^{\varepsilon, * *}\right|^{2}\right)\right) k / 2 \varepsilon} U_{j}^{\varepsilon, * *} ;
\end{aligned}
$$

where

$$
\omega_{0}=\omega_{M}=\frac{\pi}{2 M}, \quad \omega_{m}=\frac{\pi}{M}, \quad 1 \leq m \leq M-1 ; \quad \gamma_{m}=\frac{\pi}{2} c_{m}, \quad 0 \leq m \leq M .
$$

The overall time discretization error comes solely from the splitting, which is now $O\left(k^{2}\right)$ for any fixed $\varepsilon>0$.

REMARK 3.1. It is straightforward to design a fourth-order time-splitting Chebyshev-spectral (TS-Chebyshev4) method for the Schrödinger equation (3.1) similar to that in the Section 2.

REMARK 3.2. If $V(x) \equiv V=$ constant and $f(\rho) \equiv 0$ in (3.1), and $g_{1}=g_{2}=0$ in (3.2), then all the time steps in the above methods TS-Chebyshev2 and TS-Chebyshev 4 can be combined and the method can be written simply as a one step method:

$$
\begin{aligned}
& a_{l}^{\varepsilon, 0}=\frac{1}{\gamma_{l}} \sum_{m=0}^{M} U_{m}^{\varepsilon, 0} \omega_{m} T_{l}\left(\frac{x_{m}-(b+a) / 2}{(b-a) / 2}\right), \quad 0 \leq l \leq M, \\
& \mathbf{a}^{\varepsilon, n}=e^{-i V t_{n} / \varepsilon} Q(n k) \mathbf{a}^{\varepsilon, n}, \quad a_{M-1}^{\varepsilon, n}=-\sum_{\substack{m=1 \\
m \text { odd }}}^{M-2} a_{m}^{\varepsilon, n}, \quad a_{M}^{\varepsilon, n}=-\sum_{\substack{m=0 \\
m \text { even }}}^{M-2} a_{m}^{\varepsilon, n}, \\
& U_{j}^{\varepsilon, n}=\sum_{m=0}^{M} a_{m}^{\varepsilon, n} T_{m}\left(\frac{x_{j}-(b+a) / 2}{(b-a) / 2}\right), \quad 0 \leq j \leq M .
\end{aligned}
$$

This is the same as discretizing the second order space derivative in (3.1) by the Chebyshev-spectral method, and then solving the resulting ODE system exactly to $t=$ 
$t_{n}$. Therefore no time discretization error is introduced and the only error is the Chebyshev-spectral error of the spatial derivative.

When $g_{1}=g_{2}=0$ in (3.2) (e.g. $A_{\infty}=0$ in (1.4) or (1.5)), and thus $\mathbf{f}=\mathbf{0}$ in (3.10). In this case, the schemes TS-Chebyshev2 and TS-Chebyshev4 are time reversible, just as the IVP for the NLS. Furthermore, a main advantage of the method in this case is their time transverse invariance, just as it holds for the NLS itself.

4. Numerical method for vortex dynamics of NLS in 2D. For effectively computing quantized vortex dynamics of NLS in $2 \mathrm{D}$ with the non-zero far-field condition (1.5), we consider the equation $[28,23]$

$$
i u_{t}^{\varepsilon}=-\Delta u^{\varepsilon}+\frac{1}{\varepsilon^{2}}\left(\left|u^{\varepsilon}\right|^{2}-1\right) u^{\varepsilon}, \quad t>0, \quad \mathbf{x}=(x, y) \in \mathbb{R}^{2},
$$

under the nonzero far-field condition (1.5) with $A_{\infty}=1$ and initial condition (1.2). Due to the highly oscillatory nature in the transverse direction of the far-field condition (1.5) and quadratic decay rate of the solution in radial direction, it is extremely challenging to study vortex dynamics in the problem numerically. To our knowledge, there isn't numerical study of quantized vortex dynamics of the problem yet! Here we propose an efficient and accurate numerical method for the problem. The key ideas are based on the application of time-splitting techniques and the adoption of polar coordinates. Choose $R>0$ sufficient large, we truncate the problem (4.1), (1.5) into a bounded computational domain $\Omega_{R}=\left\{\mathbf{x}=(x, y)|| \mathbf{x} \mid=\sqrt{x^{2}+y^{2}}<R\right\}$ with Dirichlet boundary condition on $\Gamma=\partial \Omega$ :

$$
\begin{aligned}
& i u_{t}^{\varepsilon}=-\Delta u^{\varepsilon}+\frac{1}{\varepsilon^{2}}\left(\left|u^{\varepsilon}\right|^{2}-1\right) u^{\varepsilon}, \quad t>0, \quad \mathbf{x} \in \Omega_{R}, \\
& u^{\varepsilon}(R, \theta)=e^{i m \theta}, \quad 0 \leq \theta \leq 2 \pi .
\end{aligned}
$$

From time $t=t_{n}$ to $t=t_{n+1}$, again the NLS equation (4.2) is solved in two steps. One solves first

$$
i u_{t}^{\varepsilon}=-\Delta u^{\varepsilon}
$$

with the boundary condition (4.3) for one time step (of length $k$ ), followed by solving

$$
i u_{t}^{\varepsilon}=\frac{1}{\varepsilon^{2}}\left(\left|u^{\varepsilon}\right|^{2}-1\right) u^{\varepsilon},
$$

for the same time step. Similar to (2.5), the ODE (4.5) can be solved analytically and the solution is:

$$
u^{\varepsilon}(\mathbf{x}, t)=e^{-i\left[\left|u^{\varepsilon}\left(\mathbf{x}, t_{n}\right)\right|^{2}-1\right]\left(t-t_{n}\right) / \varepsilon^{2}} u^{\varepsilon}\left(\mathbf{x}, t_{n}\right), \quad t \in\left[t_{n}, t_{n+1}\right] .
$$

To solve (4.4), we use polar coordinates $(r, \theta)$, and discretize in the $\theta$-direction by a Fourier pseudo-spectral method, in the $r$-direction by a finite difference method and in time by a Crank-Nicholson $(\mathrm{C}-\mathrm{N})$ scheme. Assume

$$
u^{\varepsilon}(r, \theta, t)=\sum_{l=-L / 2}^{L / 2-1} \widehat{u}_{l}(r, t) e^{i l \theta},
$$


where $L$ is an even positive integer and $\widehat{u}_{l}(r, t)$ is the Fourier coefficient for the $l$ th mode. Plugging (4.7) into (4.4), noticing the orthogonality of the Fourier functions, we obtain for $-\frac{L}{2} \leq l \leq \frac{L}{2}-1$ and $0<r<R$ :

$$
\begin{aligned}
& i \partial_{t} \widehat{u}_{l}(r, t)=-\frac{1}{r} \frac{\partial}{\partial r}\left(r \frac{\partial \widehat{u}_{l}(r, t)}{\partial r}\right)+\frac{l^{2}}{2 r^{2}} \widehat{u}_{l}(r, t), \\
& \widehat{u}_{l}(R, t)=\delta_{l m}, \quad \widehat{u}_{l}(0, t)=0(\text { for } l \neq 0),
\end{aligned}
$$

where $\delta_{l m}$ is Kronecker delta. As noticed in $[19,18]$, an efficient way for discretizing (4.8)-(4.9) is to use the finite difference in space on a mesh with a shifted grid and the $\mathrm{C}-\mathrm{N}$ scheme in time. Choose an integer $M>0$, a mesh size $\Delta r=\frac{2 R}{2 M+1}$ and grid points $r_{m}=(m-1 / 2) \Delta r, m=0,1, \cdots, M+1$. Let $\hat{u}_{l, m}(t)$ be the approximation of $\hat{u}_{l}\left(r_{m}, t\right)$. A second-order finite difference discretization for (4.8)-(4.9) in space is:

$$
\begin{aligned}
& i \frac{d \hat{u}_{l, m}(t)}{d t}=-\frac{r_{m+1 / 2} \hat{u}_{l, m+1}(t)-2 r_{m} \hat{u}_{l, m}(t)+r_{m-1 / 2} \hat{u}_{l, m-1}(t)}{2 \Delta r r_{m}} \\
&+\frac{l^{2}}{2 r_{m}^{2}} \hat{u}_{l, m}(t), \quad m=1,2, \cdots, M, t_{n} \leq t \leq t_{n+1}, \\
& \hat{u}_{l, 0}(t)=(-1)^{l} \hat{u}_{l, 1}(t), \quad \hat{u}_{l, M+1}(t)=\delta_{l m}, \quad t_{n} \leq t \leq t_{n+1} .
\end{aligned}
$$

Then the ODE system (4.10)-(4.11) is discretized in time by the C-N scheme and thus only a tridiagonal linear system is to be solved which can be done in $O(M)$ arithmetic operations.

In practice, we always use the second-order Strang splitting [29], i.e. from time $t=t_{n}$ to $t=t_{n+1}$ : i) first evolve (4.5) for half time step $\Delta t / 2$ with initial data given at $t=t_{n}$; ii) then evolve (4.4) for one time step $\Delta t$ starting with the new data; iii) and evolve (4.5) for half time step $\Delta t / 2$ with the newer data.

For this algorithm, the total memory requirement is $O(M L)$ and the total computational cost per time step is $O(M L \ln L)$.

5. Numerical examples. In this section, we will study meshing strategy of the numerical methods, semiclassical limits of NLS in 1D and dynamics of quantized vortex lattice of NLS in 2D with nonzero far-field conditions.

Example 1 NLS with nonzero far-field conditions in 1D, i.e. in (1.1), we choose $d=1, V(x) \equiv 0, f(\rho)=\rho-1$. The initial condition (1.2) is always chosen in the classical WKB form:

$$
u^{\varepsilon}(x, t=0)=u_{0}^{\varepsilon}(x)=A_{0}(x) e^{i S_{0}(x) / \varepsilon}=\sqrt{\rho_{0}(x)} e^{i S_{0}(x) / \varepsilon},
$$

with $A_{0}$ and $S_{0}$ independent of $\varepsilon$, real valued, regular and with $A_{0}(x)$ and $S_{0}(x)$ decaying to $A_{\infty}$ and $x \cdot S_{\infty} /|x|$, respectively sufficiently fast as $|x| \rightarrow \infty$.

We present here computations for three types of initial values $A_{0}(x)$ and $S_{0}(x)$ :

I. Positive far-field phase $S_{\infty}=1$

$$
A_{0}(x)=1-e^{-x^{2}}, S_{0}(x)=\tanh (x), x \in \mathbb{R} ; \quad A_{\infty}=1.0, S_{\infty}=1.0 .
$$

II. Zero far-field phase $S_{\infty}=0$

$$
A_{0}(x)=1-e^{-x^{2}}, \quad S_{0}(x)=\frac{1}{\cosh (x)}, \quad x \in \mathbb{R} ; \quad A_{\infty}=1, \quad S_{\infty}=0 .
$$


III. Negative far-field phase $S_{\infty}=-1$

$$
A_{0}(x)=1-e^{-x^{2}}, S_{0}(x)=-\tanh (2 x), x \in \mathbb{R} ; \quad A_{\infty}=1.0, S_{\infty}=-1.0 .
$$

We compute with TS-Chebyshev2 (3.19) on the interval $[-16,16]$. Figure 1 pictures $\rho=\left|u^{\varepsilon}\right|^{2}$ of the solution in space-time for $\varepsilon=0.08$ under mesh size $h=\frac{1}{64}$ and time step $k=0.0001$ for the three different types data. In the figure, the quiescent and oscillatory regions are clearly recognizable and they appear to be independent of the small $\varepsilon$. The number of oscillations is proportional to $\frac{1}{\varepsilon}$, indicating that the oscillations have wavelength $O(\varepsilon)$.

To verify the weak convergence (in the $x$-variable) of $\rho^{\varepsilon}$ as $\varepsilon \rightarrow 0$, we compute the indefinite integral

$$
\int_{-\infty}^{x}\left[\rho^{\varepsilon}(s, t)-1\right] d s
$$

for various $\varepsilon$ 's. In theory, the strong convergence of the integral as $\varepsilon$ goes to zero would imply the weak convergence of $\rho^{\varepsilon}=\left|u^{\varepsilon}(x, t)\right|^{2}$ with respect to $x$. This is motivated by the works $[22,31]$ on the zero dispersion limit of the KDV equation and used for the numerical study of focusing nonlinear Schrödinger equation [3, 9, 27]. Figure 1 also displays the indefinite integral at $t=2.0$ for four different values of $\varepsilon$ : $0.64,0.32$, 0.16 and 0.08 for the three different types data. Here curves with more "corners" correspond to smaller $\varepsilon$ 's.

To test the $\varepsilon$-resolution of TS-Chebyshev2 (3.19) or TS-Chebyshev4, for each fixed $\varepsilon$, we compute the numerical solution with a very fine mesh, e.g. $h=\frac{1}{64}$, and a very small time step, e.g. $k=0.0001$, as the reference 'exact' solution $u^{\varepsilon}$. Figure 2 shows the numerical results at $t=2.0$ with $\varepsilon=0.64, k=0.01, h=\frac{1}{4} ; \varepsilon=0.16$, $k=0.005, h=\frac{1}{16} ; \varepsilon=0.08, k=0.0025, h=\frac{1}{32}$, which corresponds to the meshing strategy: $h=O(\varepsilon)$ and $k=O(\varepsilon)$, for the three different types data.

Fig. 1 clearly demonstrates the strong convergence of the indefinite integral as $\varepsilon$ goes to zero. This implies the weak convergence of $\rho^{\varepsilon}$. Furthermore Fig. 2 seems to suggest the following 'optimal' $\varepsilon$-resolution of TS-Chebyshev or TS-Cosine in order to guarantee good approximations of observables for defocusing nonlinear NLS (1.1):

$$
h=O(\varepsilon), \quad k=O(\varepsilon) .
$$

From our additional numerical results, weaker constraint on the meshing, e.g. $h=$ $O(\varepsilon)$ and $k$ independent of $\varepsilon$, gives incorrect numerical observables in this defocusing nonlinear case.

REMARK 5.1. When the TS-Chebyshev or TS-Cosine methods are applied for linear Schrödinger equation with constant/harmonic oscillator potential, e.g. Examples 1\&2 in [2], we have observed the following meshing strategy: $k$-independent of $\varepsilon$ and $h=O(\varepsilon)$, which is the same as those for time-splitting Fourier-spectral or Sine-spectral methods [2, 3]. We omitted the details here.

Example 2 Dynamics of quantized vortex lattice of NLS (4.1) in 2D with the nonzero far-field conditions (1.5). The initial condition (1.2) is chosen as

$$
u^{\varepsilon}(\mathbf{x}, t=0)=u_{0}^{\varepsilon}(\mathbf{x})=\prod_{j=1}^{N} \phi_{n_{j}}\left(\mathbf{x}-\mathbf{x}_{j}\right), \quad \mathbf{x} \in \mathbb{R}^{2},
$$


a)
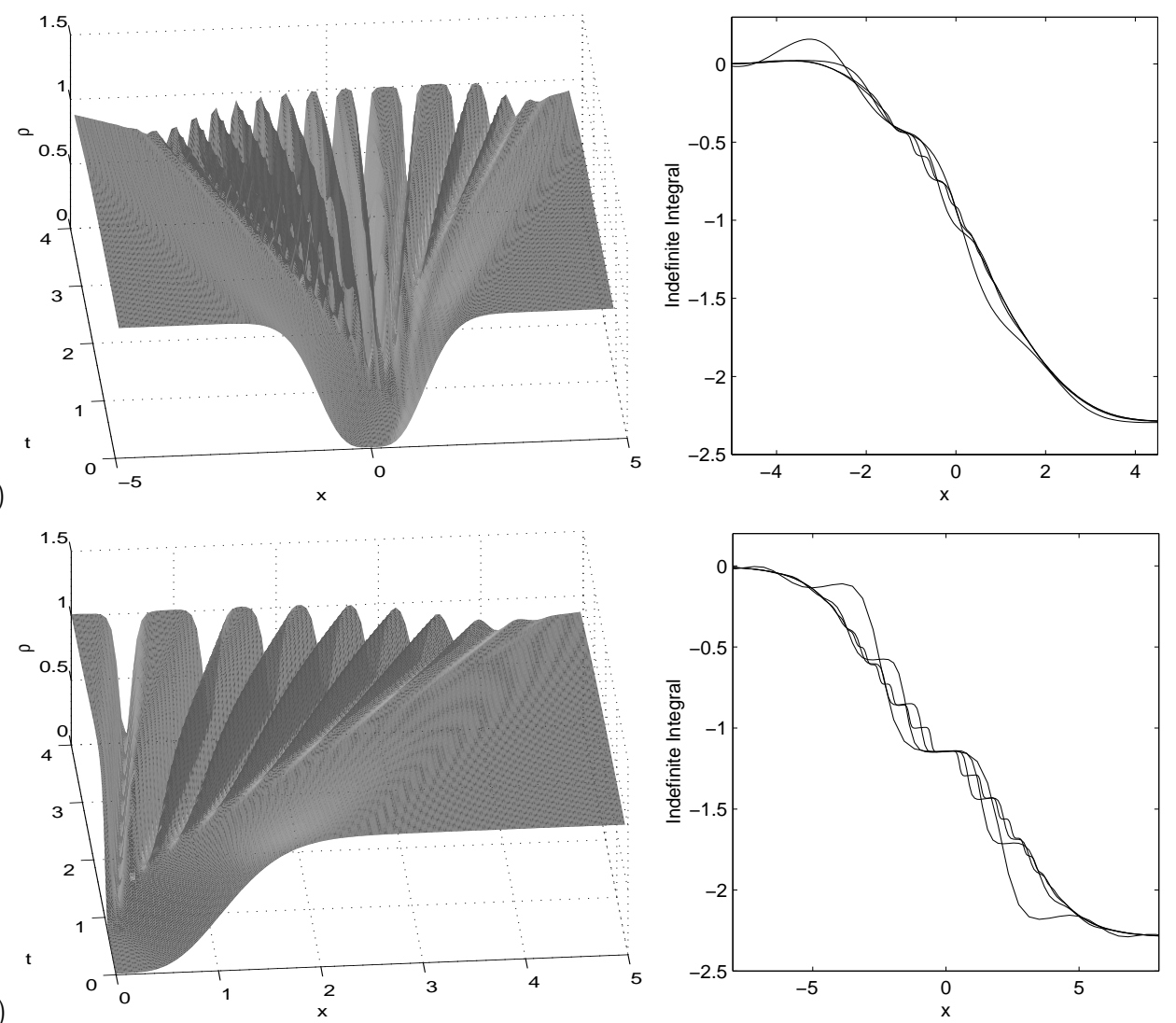

b)
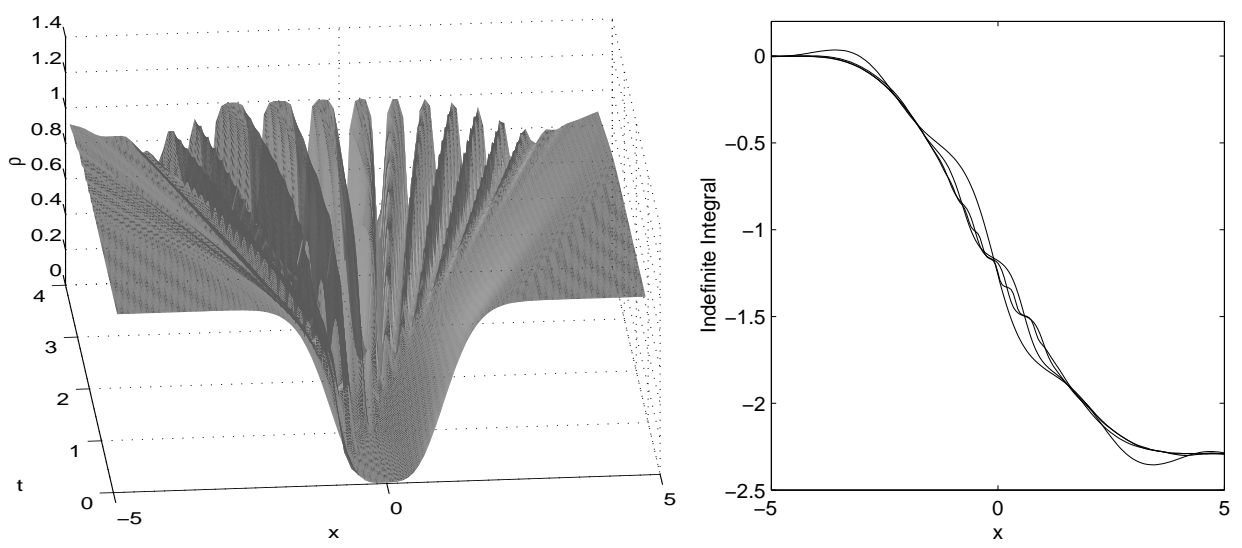

FIG. 1. Evolution of the position density for $\varepsilon=0.08$ (left column) and indefinite integrals of the density $\rho^{\varepsilon}(x, t=2.0)-1$ for $\varepsilon=0.64,0.32,0.16$ and 0.08 (right column), illustrating weak convergence (curves with more "corners" correspond to smaller $\varepsilon$ 's), in Example 1 under mesh size $h=\frac{1}{64}, k=0.0001$. a) Type $I$ (5.2) with $S_{\infty}=1.0 ;$ b) type $I I(5.3)$ with $S_{\infty}=0.0 ;$ c) type III (5.4) with $S_{\infty}=-1.0$.

where $\phi_{n}(\mathbf{x})=f_{n}(r) e^{i n \theta}$ is a stationary central vortex state of (4.1) with winding 
position density

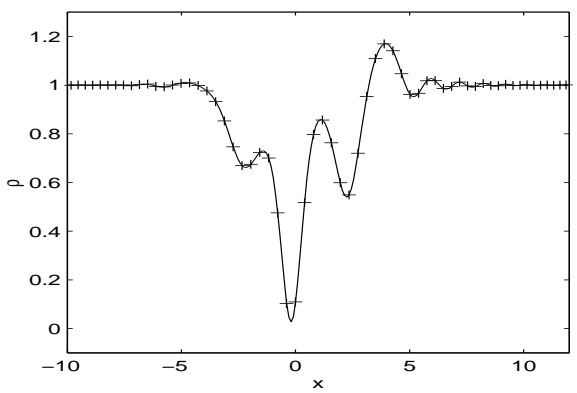

a).

b).

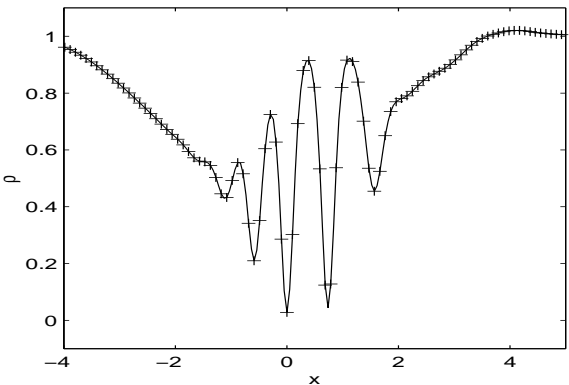

c).

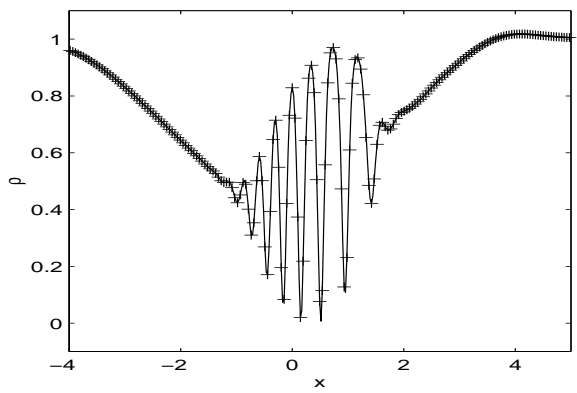

current density
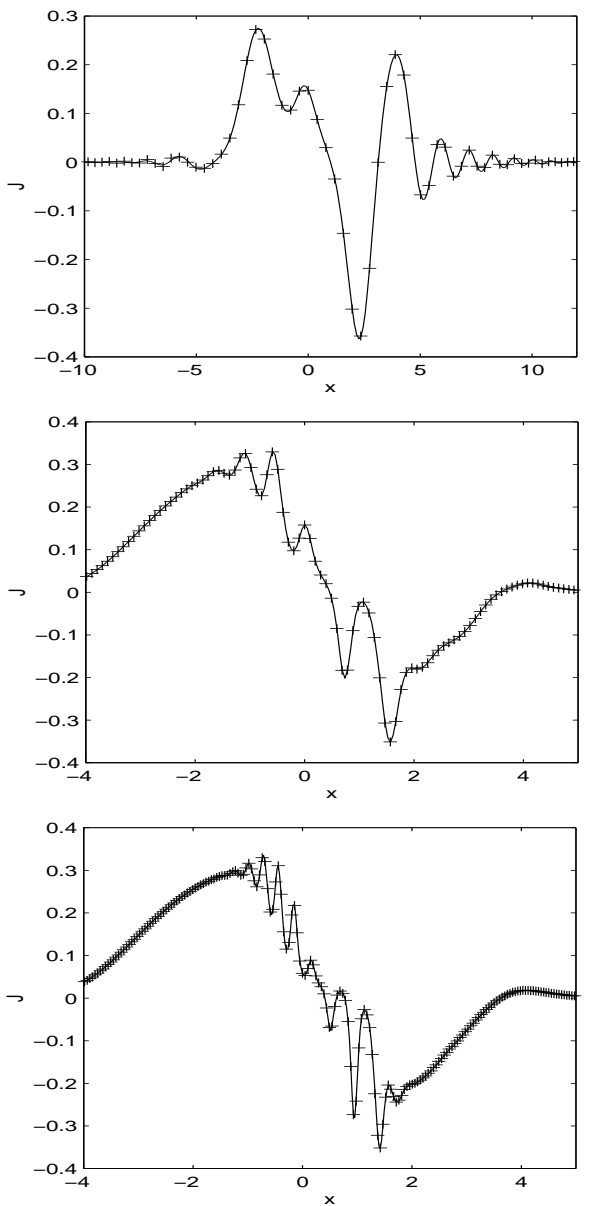

FIG. 2. Numerical solutions at $t=2.0$ in Example 1 for NLS by using TS-Cosine4 (2.12) or TS-Chebyshev4. '-': 'exact' solution, ' +++ ': numerical solution. a). $\varepsilon=0.64, k=0.01, h=\frac{1}{4}$, b). $\left.\varepsilon=0.16, k=0.0025, h=\frac{1}{16}, c\right) . \varepsilon=0.08, k=0.00125, h=\frac{1}{32}$. Here $h=O(\varepsilon), k=O(\varepsilon)$. (i) Type $I$ (5.2) with $S_{\infty}=1.0$.,

number (or index) $n \neq 0$, i.e. $f_{n}(r)$ satisfies [28]

$$
\begin{aligned}
& -\frac{1}{r} \frac{d}{d r}\left(r \frac{d f_{n}(r)}{d r}\right)+\frac{n^{2}}{r^{2}} f_{n}(r)+\frac{1}{\varepsilon^{2}}\left(f_{n}^{2}(r)-1\right) f_{n}(r)=0,0<r<\infty, \\
& f_{n}(0)=0, \quad f_{n}(\infty)=1 .
\end{aligned}
$$

The nonzero far-field condition (1.5) is chosen as $m=\sum_{j=1}^{N} n_{j}$.

Here we present dynamics of quantized vortex lattice for two types of initial data with $\varepsilon=1$ in (5.7) and (4.1):

Case I. We take $N=5, n_{1}=n_{2}=n_{3}=n_{4}=n_{5}=1, \mathbf{x}_{1}=(0,0)^{T}, \mathbf{x}_{2}=(4,0)^{T}$, $\mathbf{x}_{3}=(-4,0)^{T}, \mathbf{x}_{4}=(0,4)^{T}, \mathbf{x}_{5}=(0,-4)^{T}$ in $(5.6)$. 
position density

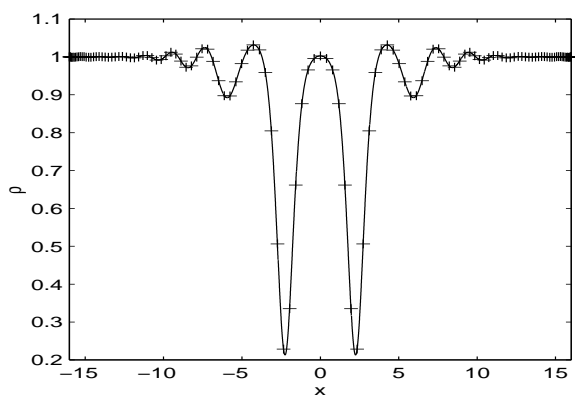

a).

b).

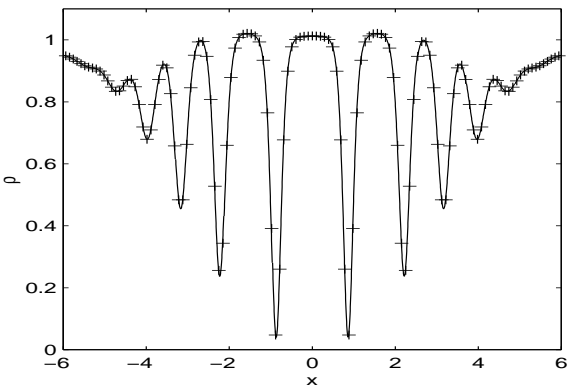

c).

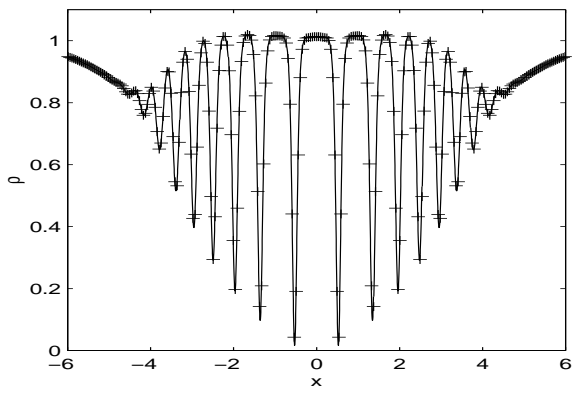

current density
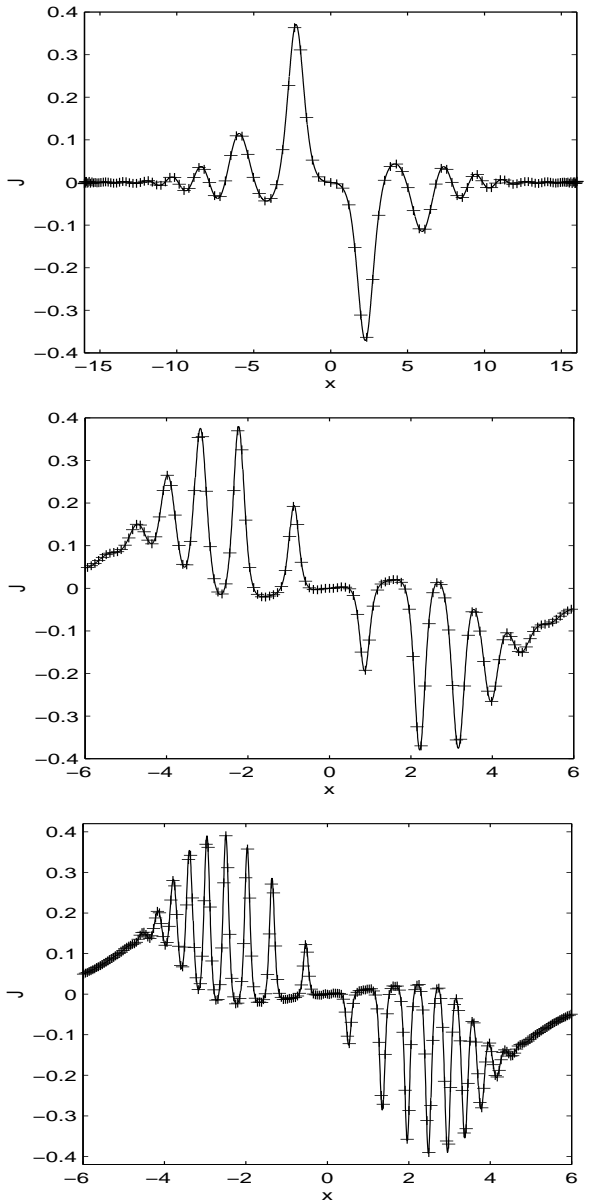

FIG. 2 (CONT'D). (ii) Type II (5.3)with $S_{\infty}=0.0$.

Case II. We take $N=4, n_{1}=n_{2}=1, n_{3}=n_{4}=-1, \mathbf{x}_{1}=(2,0)^{T}, \mathbf{x}_{2}=(-2,0)^{T}$, $\mathbf{x}_{3}=(0,2)^{T}, \mathbf{x}_{4}=(0,-2)^{T}$ in $(5.6)$.

We compute this problem with the method proposed in section 4 on a disk with radius $R=200$. The mesh sizes and time step are chosen as $\Delta r=1 / 56, \Delta \theta=\pi / 128$ and $\Delta t=0.0001$. To quantify the numerical results, we define the hydrodynamic velocity

$$
\mathbf{u}=(u, v)=\frac{\bar{u}^{\varepsilon} \nabla u^{\varepsilon}-u^{\varepsilon} \nabla \bar{u}^{\varepsilon}}{2 i\left|u^{\varepsilon}\right|^{2}} .
$$

Figure 3 plots the velocity fields for dynamics of quantized vortex lattices in cases I\&II. Figure 4 shows surfaces of the density function $\left|u^{\varepsilon}\right|^{2}$ at different times. Furthermore, Figure 5 plots the trajectory of the vortex centers.

From Figs. 3-5, we can draw the following conclusions for dynamics of quantized vortex lattice: (i) In case I, the vortex initially located at the origin does not move at 
position density

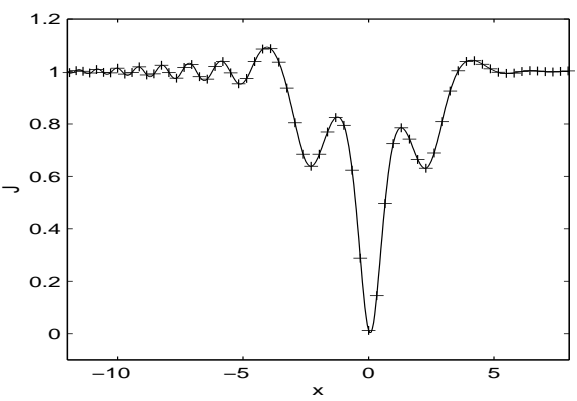

a).

b).

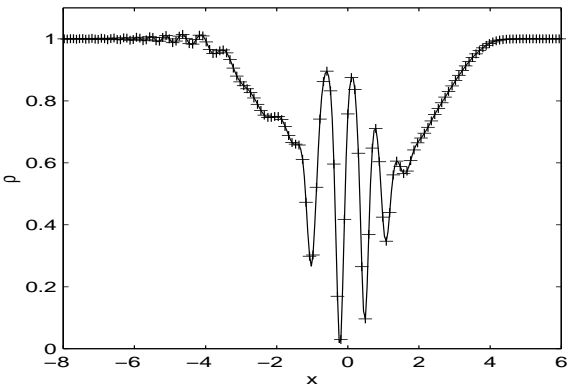

c).

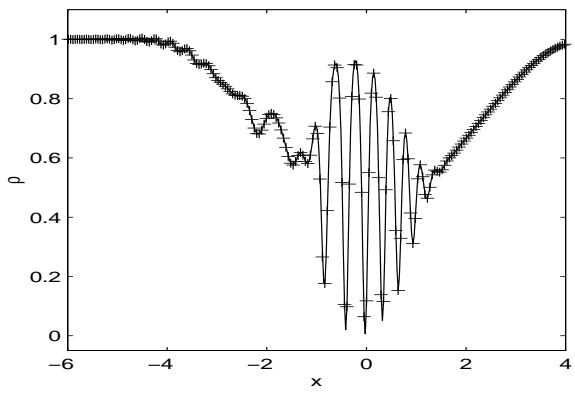

current density
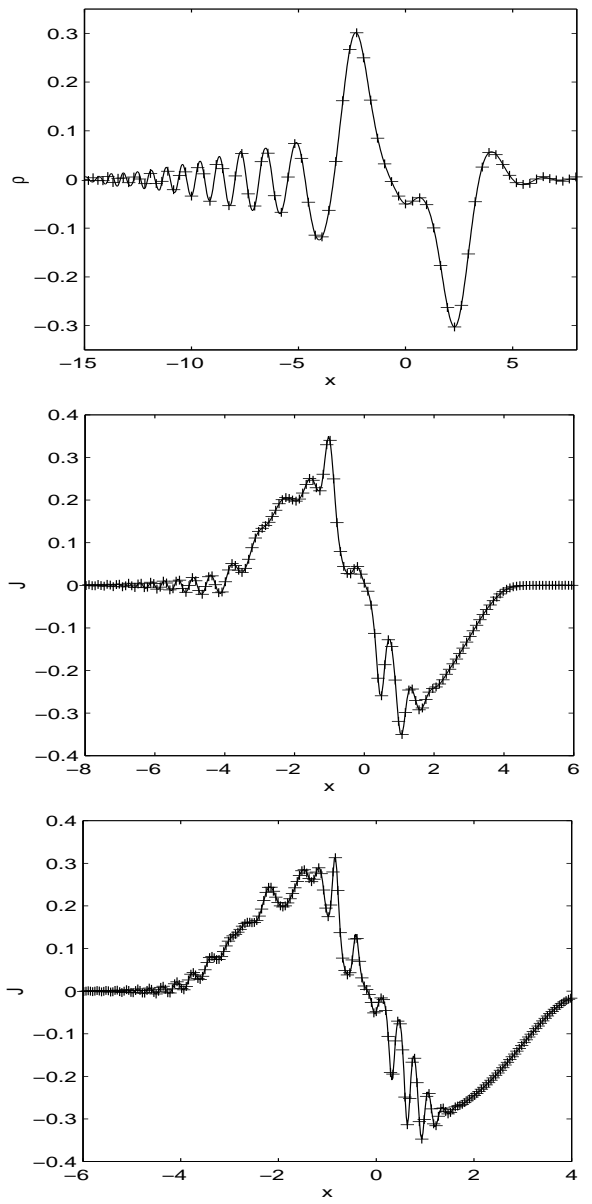

FIG. 2 (CONT'D). (iii) Type III (5.4) with $S_{\infty}=-1.0$.

all which is due to the symmetric setup of the initial condition (cf. Figs. 3i,4i\&5a), the other four vortices rotate around the origin and they move along a circle after some time (cf. Figs. 3i,4i\&5a). (ii). In case II, the vortex initially located at $(2,0)$ with winding number $m=1$ will merges with the vortex initially located at $(0,-2)$ with winding number $m=-1$, the other two vortices with opposite winding number will collide too (cf. Figs. 3ii,4ii\&5b). After each two vortices with opposite winding number collide, a shock wave is generated (cf. Fig. 3ii\&4ii) and no vortex is left in the density function. For more numerical study of quantized vortex stability and interaction of Ginzburg-Landau-Schrödinger equation, we refer to [1].

6. Conclusions. Some new numerical methods are presented for the nonlinear Schrödinger equations in the semiclassical regimes with nonzero far-field conditions. The TS-Cosine method is proposed when the nonzero far-field conditions are or can be reduced to homogeneous Neumann conditions, the TS-Chebyshev method is presented for more general nonzero far-field conditions other than homogeneous Neumann 

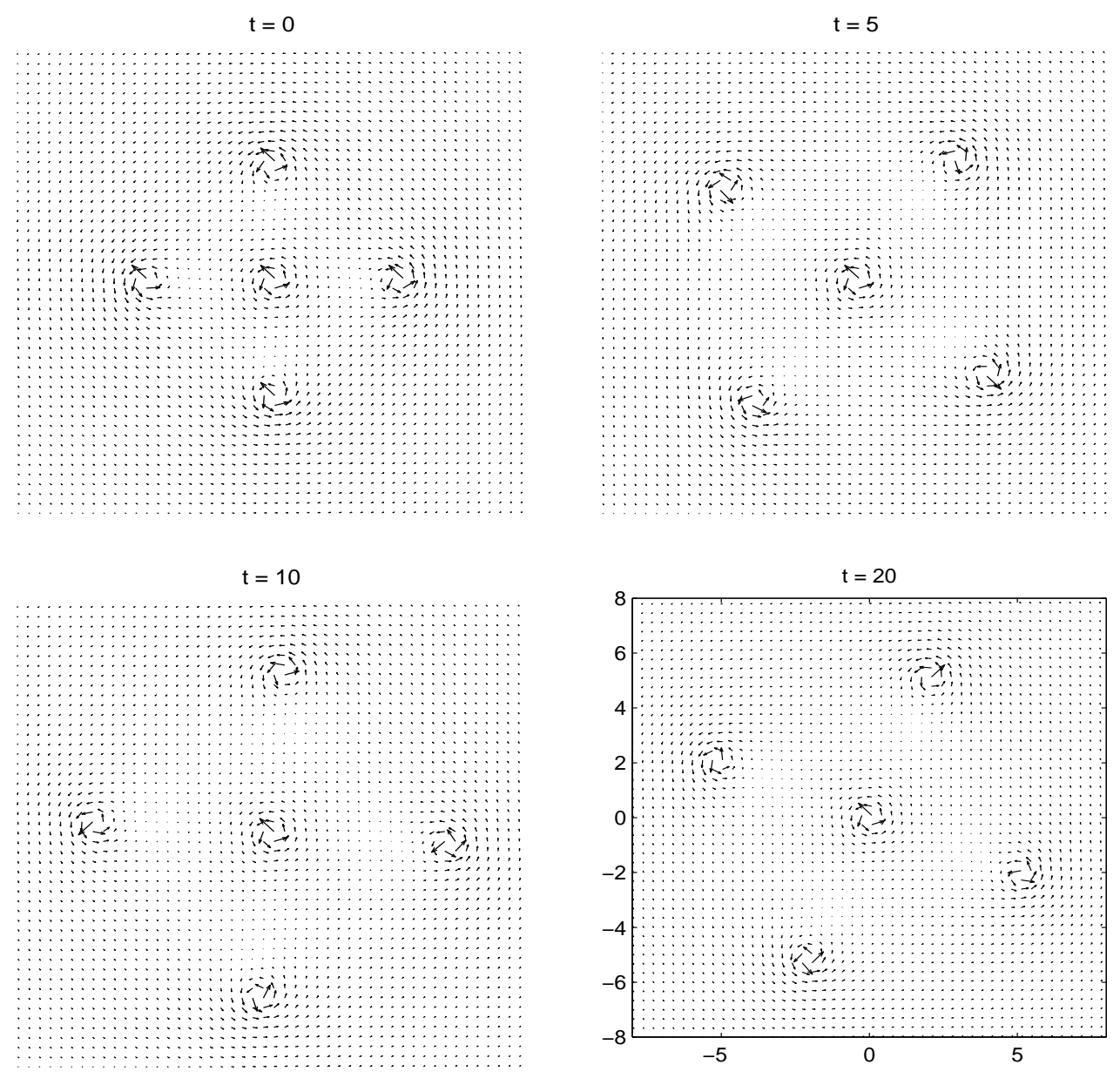

FIG. 3. Velocity fields for dynamics of quantized vortex lattices in Example 2. (i) For case I.

conditions, and an efficient and accurate method is proposed for studying dynamics of quantized vortex of NLS in 2D with nonzero far-field conditions. The TS-Cosine method is explicit, unconditionally stable, time reversible, time-transverse invariant, spectral accuracy in space and conserves the position density, where the TS-Chebyshev method can deal with more general nonzero far-field conditions, and is explicit, time reversible and time-transverse invariant when it is applied to NLS with zero far-field conditions. After applying a diagonalization technique for the ODE system (3.10) in phase space of the TS-Chebyshev method, we observe 'optimal' $\varepsilon$-resolution of the two methods for obtaining 'correct' physical observables for NLS with non-zero farfield conditions in the semi-classical regimes: time step $k$-independent of $\varepsilon$ and mesh size $h=O(\varepsilon)$ for linear case; $k=O(\varepsilon)$ and $h=O(\varepsilon)$ for defocusing nonlinear case. The methods are applied to study semiclassical limits of NLS in 1D and dynamics of quantized vortex lattice of NLS in 2D with nonzero far-field conditions. Comparing the methods, the TS-Cosine method is more computational efficient and less memory required than the TS-Chebyshev method, but the latter can deal with more general nonzero far-field conditions. From our extensive numerical experiments in $[2,3]$ and 

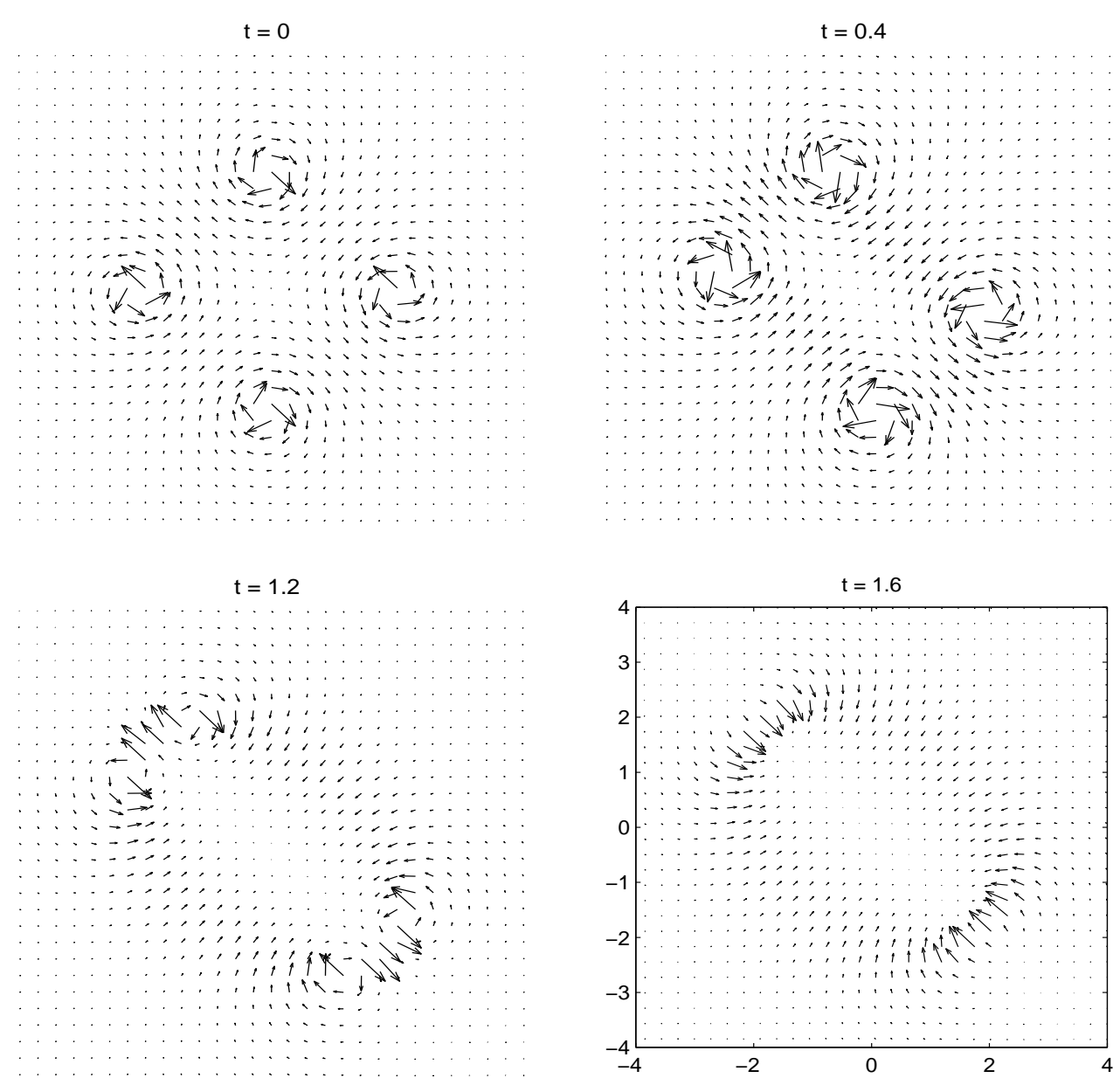

Fig. 3 (CONT'D). (ii) For case II.

in this paper: For NLS with zero far-field conditions, we recommend to use the timesplitting Fourier-Spectral (TS-Fourier) [2, 3] or Sine-spectral (TS-Sine) [5] method; for NLS with nonzero far-field conditions which are and can be reduced to homogeneous Neumann far-field conditions, we recommend to use the TS-Cosine method; and for NLS with other more general nonzero far-field conditions, we recommend to use the TS-Chebyshev method.

Acknowledgment. This work was supported by the National University of Singapore grant No. R-151-000-030-112. The author thanks Miss Yanzhi Zhang for her help in some calculations of Example 2.

\section{REFERENCES}

[1] W. BaO, Q. Du AND Y. Zhang, Numerical simulation of vortex dynamics of Ginzburg-LandauSchrödinger equation, in preparation.

[2] W. BaO, Shi Jin And P.A. Markowich, On time-splitting spectral approximations for the Schrödinger equation in the semiclassical regime, J. Comput. Phys., 175 (2002), pp. 487524 . 

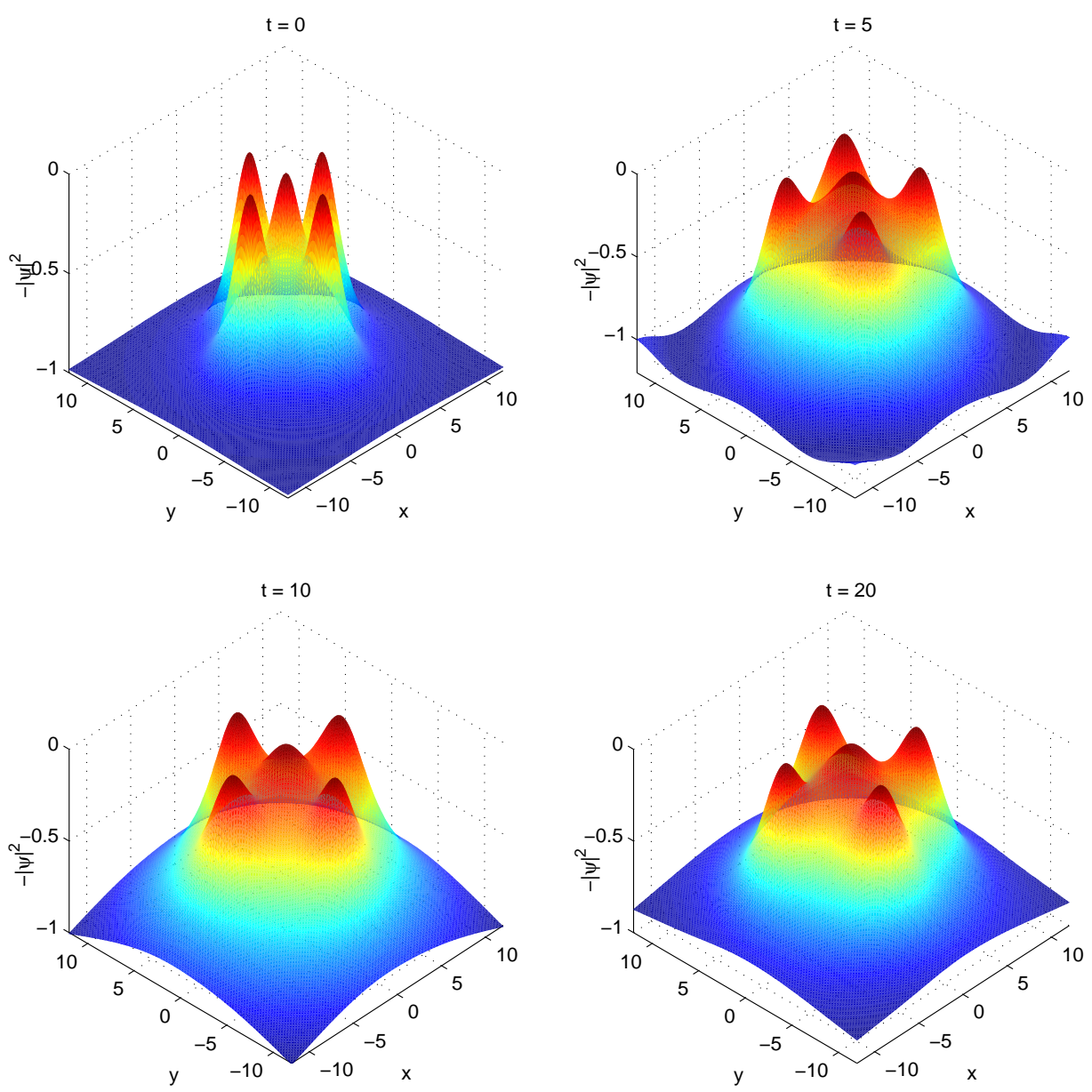

FIG. 4. Surface plots of density function $\left|u^{\varepsilon}\right|^{2}$ (labelled as $|\psi|^{2}$ ) for dynamics of quantized vortex lattices in Example 2. (i) For case I.

[3] W. BaO, Shi Jin And P.A. Markowich, Numerical study of time-splitting spectral discretizations of nonlinear Schrödinger equations in the semi-classical regimes, SIAM J. Sci. Comput., 25 (2003), pp. 27-64.

[4] W. Bao, D. Jaksch And P.A. Markowich, Numerical solution of the Gross-Pitaevskii equation for Bose-Einstein condensation, J. Comput. Phys., 187 (2003), pp. 318-342.

[5] W. BAO, D. JAKSCH, An explicit unconditionally stable numerical methods for solving damped nonlinear Schrodinger equations with a focusing nonlinearity, SIAM J. Numer. Anal., 41 (2003), pp. 1406-1426.

[6] J.C. Bronski and D. W. McLaughuin, Semiclassical behavior in the NLS equation: optical shocks - focusing instabilities, Singular Limits of Dispersive Waves, Plenum Press, New York and London, 1994.

[7] C. Canuto, M.Y. Hussaini, A. Quarteroni and T.A. Zhang, Spectral Methods in Fluid Dynamics, Springer-Verlag, Berlin, 1988.

[8] R. CARles, Remarques sur les mesures de Wigner, preprint.

[9] H.D. Ceniceros and F.R. Tian, A numerical study of the semi-classical limit of the focusing nonlinear Schrödinger equation, Phys. Lett. A., 306 (2002), pp. 25-34.

[10] J.E. Colliander and R.L. Jerrard, Vortex dynamics for the Ginzburg-Landau-Schrödinger equation, IMRN Int. Math. Res. Notices, 7 (1998), pp. 333-358.

[11] G. Fibich and G. Papanicolaou, Self-focusing in the perturbed and unperturbed nonlinear Schrödinger equation in critical dimension, SIAM J. Appl. Math., 60 (2000), pp. 183-240. 

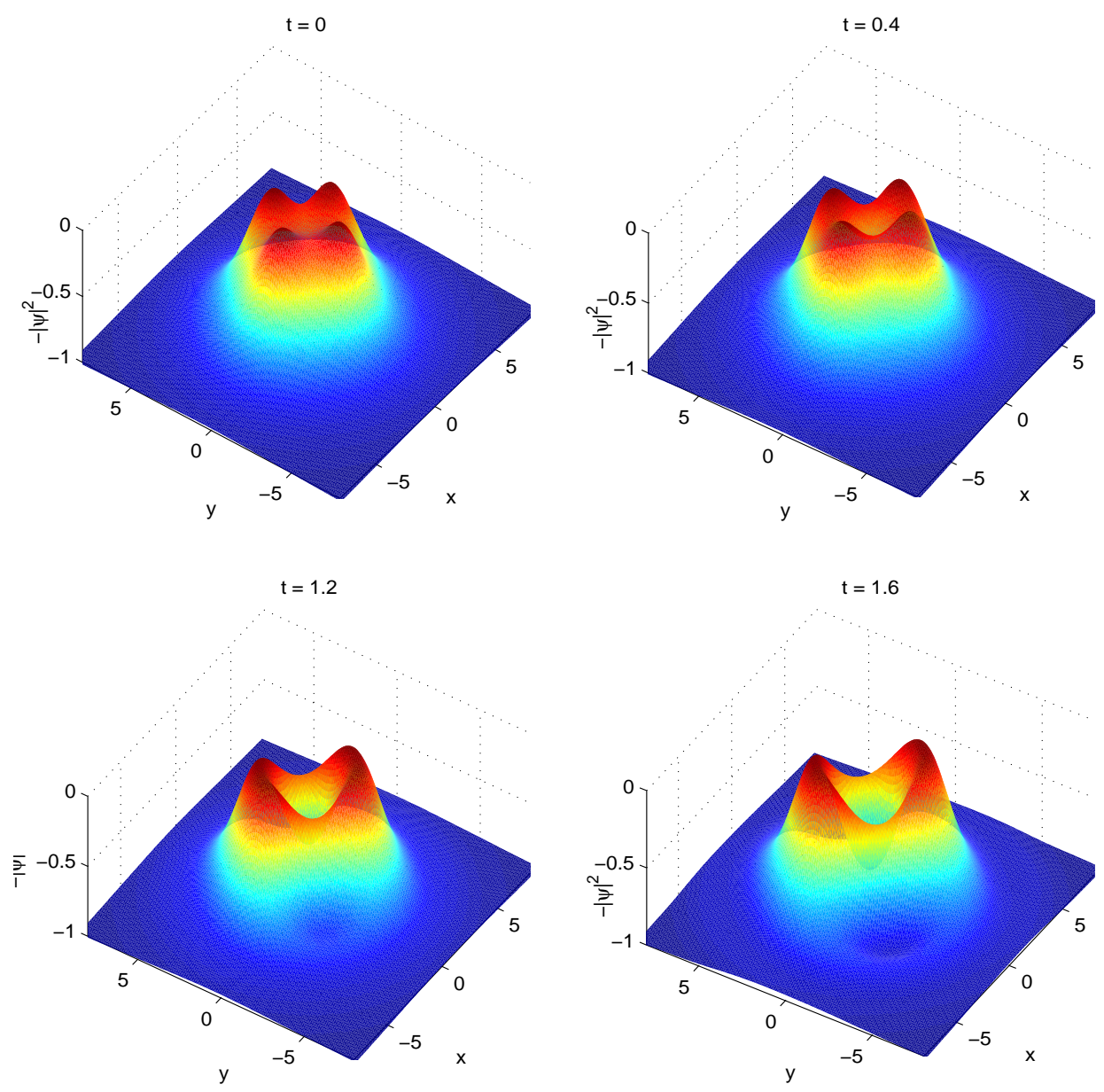

FIG. 4 (CONT'D). (ii) For case II.

[12] I. Gasser And P.A. Markowich, Quantum hydrodynamics, Wigner transforms and the classical limit, Asymptotic Analysis, 14 (1997), pp. 97-116.

[13] P. GÉrard, Microlocal defect measures, Comm. PDE., 16 (1991), pp. 1761-1794.

[14] P. Gérard, P.A. Markowich, N.J. Mauser and F. Poupaud, Homogenization limits and Wigner transforms, Comm. Pure Appl. Math., 50 (1997), pp. 321-377.

[15] D. Gottlieb And S.A. Orszag, Numerical Analysis of Spectral Methods, SIAM, Philadelphia, 1977.

[16] Shan Jin, C.D. Levermore and D. W. McLaughlin, The semiclassical limit of the defocusing NLS hierarchy, Comm. Pure Appl. Math., LII (1999), pp. 613-654.

[17] Shan Jin, C.D. Levermore And D. W. McLaughlin, The behavior of solutions of the NLS equation in the semiclassical limit, Singular Limits of Dispersive Waves, Plenum Press, New York and London, 1994.

[18] M.-C. LAI, W.-W. LIN AND W. WANG, A fast spectral/difference method without pole conditions for Poisson-type equations in cylindrical and spherical geometries, IMA J. of Numer. Anal., 22 (2002), pp. 537-548.

[19] M.-C. LAI AND W.-C. WANG, Fast direct solvers for Poisson equation on 2D polar and spherical geometries, Numer. Methods Partial Differential Eq., 18 (2002), pp. 56-68.

[20] M.J. Landman, G. Papanicolaou, C. Sulem, P.L. Sulem and X.P. Wang, Stability of isotropic singularities for the nonlinear Schrödinger equation, Phys. D, 47 (1991), pp. $393-415$.

[21] Laudau and Lifschitz, Quantum Mechanics: non-relativistic theory, Pergamon Press, New 


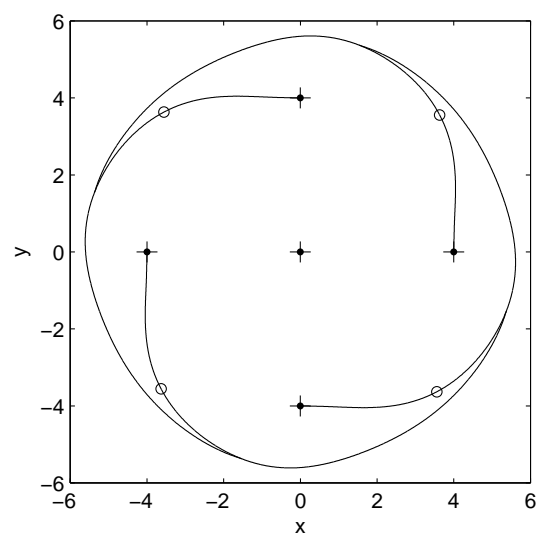

a)

FIG. 5. Trajectory of the vortex centers for dynamics of quantized vortex lattices in Example 2. For case I ( $c f$. a )) and case II ( $c f . b)$ ). ' + ': for winding number $m=1$; '-': for winding number $m=-1$; 'o': merging position in $b$ ).

York, 1977.

[22] P.D. Lax And C.D. Levermore, The small dispersion limit for the Kortewegde Vires Equation I, II and III, Comm. Pure Appl. Math. 36 (1983), pp. 253-290, pp. 571-593, pp. 809-830.

[23] F-H Lin AND J.X. XIN, On the incompressible fluid limit and the vortex motion law of the nolinear Schrödinger equation, Comm. Math. Phys., 200 (1999), pp. 249-274.

[24] P.A. Markowich, N.J. Mauser and F. Poupaud, A Wigner function approach to semiclassical limits: electrons in a periodic potential, J. Math. Phys., 35 (1994), pp. 1066-1094.

[25] P.A. Markowich, P. Pietra and C. Pohl, Numerical approximation of quadratic observables of Schrödinger-type equations in the semi-classical limit, Numer. Math., 81 (1999), pp. $595-630$.

[26] P.A. Markowich, P. Pietra, C. Pohl and H.P. Stimming, A Wigner-Measure Analysis of the Dufort-Frankel scheme for the Schrödinger equation, SIAM J. Numer. Anal., 40 (2002), pp. 1281-1310.

[27] P.D. Miller, S. Kamvissis, On the semiclassical limit of the focusing nonlinear Schrödinger equation, Phys. Letters A, 247 (1998), pp. 75-86.

[28] J.C. Neu, Vortices in complex scalar fields, Physica D, 43 (1990), pp. 385-406.

[29] G. Strang, On the construction and comparison of difference schemes, SIAM J. Numer. Anal., 5 (1968), pp. 505-517.

[30] L. TARTAR, H-measures: a new approach for studying homogenization, oscillations and concentration effects in partial differential equations, Proc. Roy. Soc. Edinburgh Sect. A, 115 (1990), pp. 193-230.

[31] G.B. Venakides, The zreo dispersion limit of the periodic KDV equation, AMS Transaction, 301 (1987), pp. 189-226.

[32] V.E. Zakharov and A.B. Shabat, Exact theory of of two-dimensional self-focusing and onedimensional self-modulation of waves in nonlinear media, Sov. Phys. JETP, 34 (1972), pp. $62-69$.

[33] V.E. Zakharov and A.B. Shabat, Interaction between solitons in a stable medium, Sov. Phys. JETP, 37 (1973), pp. 823-828.

[34] H. YoshidA, Construction of higher order symplectic integrators, Phys. Lett. A, 150 (1990), pp. 262-268. 
W. BAO 